\title{
EQUILIBRIUM POINTS OF NON-COOPERATIVE \\ RANDOM AND BAYESIAN GAMES
}

by

Nicholas C. Yannelis

Discussion Paper No. 241, August, 1987

\author{
Center for Economic Research \\ Department of Economics \\ University of Minnesota \\ Minneapolis, Minn 55455
}

* Department of Economics, University of Minnesota, Minneapolis, MN 55455.

Department of Economics, Tulane University, New Orleans, LA 70118 (after August 15, 1987).

** I would like to thank Erik Balder, M. All Khan and Aldo Rustichini for several useful discussions. An earlier version of this paper was circulated in July of 1986 under the title "Non-Cooperative Random Games." 
Abstract: We provide random equilibrium existence theorems for noncooperative random games with a countable number of players. Our results give as corollaries generalized random versions of the ordinary equilibrium existence result of Nash [18]. Moreover, they can be used to obtain equilibrium existence results for games with incomplete information, and in particular Bayesian games. In view of recent work on applications of Bayesian games and Bayesian equilibria, the latter results seem to be quite useful since they delineate conditions under which such equilibria exist. 


\section{INTRODUCTION}

A finite game consists of a set of players $I=\{1,2, \ldots, n\}$ each of whom is characterized by a strategy set $X_{i}$ and a payoff (utility) function $u_{i}: \prod_{j} \in X_{j} \rightarrow \mathbb{R}$. An equilibrium for this game is a strategy vector such that no player can increase his/her payoff by deviating from his/her equilibrium strategy, given that the other players use their equilibrium strategies, i.e.,

$x^{*} \in \prod_{1 \in I} x_{1}$ is an equilibrium if $u_{1}\left(x^{*}\right)=\max _{y_{i} \in x_{i}} u_{i}\left(x_{1}^{*}, \ldots, x_{1-1}^{*}, y_{i}, x_{i+1}^{*}, \ldots, x_{n}^{*}\right)$ for all $1 \in I$. The above game form and the notion of equilibrium were both introduced in a seminal paper by Nash [18]. ${ }^{1}$ In that same paper Nash proved by means of the Brouwer fixed point theorem, the existence of an equilibrium for the above game, where strategy sets were subsets of $\mathbb{R}^{\ell}$, 1.e., the $\ell$-fold product of the real numbers. The work of Nash has found very interesting applications in Game Theory and Mathematical Economics (see for instance Arrow-Debreu [1] or Debreu [6] among others). Generalizations of Nash's equilibrium existence theorem to games where strategy sets were subsets of arbitrary Hausdorff linear topological spaces, were obtained by Fan [9] and Browder [4] among others. The results of Fan and Browder were proved by means of infinite dimensional fixed point theorems. Subsequently to the above work, research in economics (see for instance Shafer-Sonnenschein [24]) necessitated further generalizations of Nash's equilibrium existence result, to games where each player is equipped with a preference correspondence (instead of a payoff function), which need not be transitive or complete and therefore need not be representable by a utility function. The latter work was motivated by empirical results which indicated that in many instances agents' behavior is not necessarily transitive.

A common characteristic of all the above results is that they are deterministic, 1.e., players cannot accommodate any kind of uncertainty or randomess in their responses to potential 
changes in their primitive environment. In reality however, there are many factors which go beyond the control of players and cannot be influenced by their actions. In that sense, it seems natural to assume that player's payoff functions depend not only on the strategies, but on the states of nature of the world as well. In other words payoff functions can be random. This is the type of the game we will consider in this paper. Of course with random payof functions the equilibrium strategy vector will be random as well, and therefore the equilibrium will change from one state of the environment to another.

It is the purpose of this paper to prove random equilibrium existence results for a quite general form of random games. In particular, as in Shafer-Sonnenschein [23] or Yannelis-Prabhakar [24] instead of assigning each player a random payoff or utility function, we equip each player with a random preference correspondence which need not be representable by a random utility function. It should be noted, however, that our random equilibrium results, provide as corollaries random versions of the theorems of Nash, Fan and Browder. Moreover, we show that these random equilibrium theorems can be used to obtain equilibrium existence results for games with incomplete information, and in particular, for Bayesian games. The main reference for the latter type of games appears to be Harsanyi's [10] seminal paper. Recently there is a growing literature on this subject. In particular, Bayesian games have found very interesting applications in economic theory, e.g., Aumann [2], Myerson [17], Palfrey-Srivastava [19, 20], Peck-She11 [21] and Postlewaite-Schmeidler [22] among others. ${ }^{2}$

As in $[2,10,17,19,20,21]$ by the term Bayesian games we mean games, where each plaver $i$ is characterized by a strategy set $x_{i}$, a random utility function $h_{i}$ defined on the product space $\Omega \times X$ (where $\Omega$ is the set of states of the world and $x=\prod_{i \in I} x_{i}$ ), an information set $s_{i}$ (where $S_{i}$ is a partition of $\Omega$ ), and a prior $q_{i}$ (i.e., a probability measure on $\Omega)$. In this setting the corresponding natural extension of Nash's 
equilibrium concept is that of a Bayesian equilibrium. In particular, if we denote by $E_{1}(w)$ the event in $S_{1}$ containing the realized state of nature ${ }_{\omega} \in \Omega$, then each agent will choose a strategy which maximizes expected utility conditional on his/her own event $E_{1}(\omega)$.

Note that in this Bayesian game the conditional expected utility of each player is a random function, i.e., depends on the states of nature of the world and on the strategles. Hence, in essence the problem of the existence of a Bayestan equilibrium is converted to a random equilibrium existence problem, simply by thinking of the conditional expected utility of each player as his/her random payoff function of some random game. It is exactly for this reason that the existence of a Bayesian equilibrium for a Bayesian game follows directly from the existence of a random equilibrium for a random game. The latter result seems to be quite interesting. Specifically, in view of recent work on Bayesian games and Bayesian equilibrlum, e.g., Palfrey-Srivastava [19, 20] Peck-Shel1 [21] and Postlewaite-Schmeidler [22], among others, it is useful to delineate conditions under which such equilibria exist.

Finally, we would like to note that the proofs of our random equilibrium existence theorems are not based on any of the ordinary equilibrium existence results of Nash or Fan or Browder. Our arguments start from a rudimentary level and provide a different way to prove the deterministic results of the above authors. As the deterministic results of Nash, Fan. and Browder are based on deterministic fixed point theorems, the proofs of our random equilibrium existence theorems are based on random fixed points. The idea behind the need of a random fixed point can be intultively grasped simply by noting that with random payoff functions the best reply correspondence becomes random as well, and therefore a random extension of the Kakutani-Fan-Glicksberg fixed point theorem seems to be required. To this end, we prove a random version of Fan's [8, Theorem 6, p. 238] coincidence theorem, which gives as corollary a random version of the KakutaniFan-Glicksberg fixed point theorem. In addition we employ Aumann-type 
measurable selection theorems and some recent Caratheodory-type selections results proved in Kim-Prikry and the author $[12,13]$.

The paper is organized as follows: Section 2 contains several preIfminary results of measure theoretic character. Moreover, a random version of Fan's coincidence theorem is established. The main results of the paper are stated in Section 3 and their proofs are gathered in Section 4. Section 5 contains some discussion of the related literature on games with incomplete information. Finally some concluding remarks are given in Section 6 . 


\section{PRELIMINARIES}

\subsection{Notation}

$2^{A}$ denotes the set of all subsets of the set $A$

conA denotes the convex hull of the set A

1 denotes the set theoretic subtraction

$\mathbb{R}^{\ell}$ denotes the $\ell$-fold Cartesian product of the set of real numbers $\mathbb{R}$

proj denotes projection

If $\phi: X \rightarrow 2^{Y}$ is a correspondence, then $\left.\phi\right|_{U}: U \rightarrow 2^{Y}$ denotes the restriction of $\phi$ to $U$

$\phi \quad$ denotes the empty set.

\subsection{Upper and Lower Semicontinuous Correspondences}

Let $\mathrm{X}$ and $\mathrm{Y}$ be sets. The graph $G_{\phi}$ of a correspondence $\phi: X \rightarrow 2^{Y}$ is the set $G_{\phi}=\{(x, y) \in X \times Y: y \in \phi(x)\}$. If $X$ and $Y$ are topological spaces, $\phi: X \rightarrow 2^{Y}$ is said to have an open graph if the set $G_{\phi}$ is open in $X \times Y$; $\phi$ : $X \rightarrow 2^{Y}$ is said to be lower semicontinuous (I.s.c.) if the set $\{x \in X: \phi(x) \cap V \neq \phi\}$ is open $i n X$ for every open subset $V$ of $Y$ and; $\phi: X \rightarrow 2^{Y}$ is said to be uppersemicontinuous (u.s.c.) if the set $\{x \in X: \phi(x) \subset v\}$ is open in $X$ for every open subset $\mathrm{V}$ of $\mathrm{Y}$. It can be easily checked that if a correspondence has an open graph, then it is 1.s.c., but the reverse is not true, (see [25, p. 237]).

We will need the following facts.

(F.2.1) Let $X$ be a topological space and $Y$ be a linear topological space. If the correspondence $\phi: X \rightarrow 2^{Y}$ is 1.s.c. then the correspondence $\psi: X \rightarrow 2^{Y}$ defined by $\psi(x)=\operatorname{con} \phi(x)$ is also 1.s.c. [14, Proposition 3.6, p. 366]. 
(F.2.2) Let $X$ be a topological space and $\left\{Y_{i}: i \in I\right\}$, ( $I$ can be any finite or infinite set) be a family of compact spaces. Let $Y=\prod_{i \in T_{i}}$. If for each $i \in I$, the correspondence $F_{i}: X \rightarrow 2^{Y_{1}}$ is u.s.c. and closed valued then the correspondence $F: X \rightarrow 2^{Y}$ defined by $F(x)=\prod_{i \in I_{i}} F_{i}$ is also u.s.c. [ 7, Lemma 3, p. 124].

\subsection{Auxiliary Measure Theoretic Facts}

Let $X, Y$ be topological spaces and $\phi: X \rightarrow 2^{Y}$ be a nonempty valued correspondence. A continuous selection for $\phi$ is a continuous function $f: X \rightarrow Y$ such that $f(x) \in \phi(x)$ for all $x \in X$.

Let $(\Omega, Q)$ be a measurable space, $Y$ be a topological space and $\phi: \Omega \rightarrow 2^{Y}$ be a nonempty-valued correspondence. A measurable selection for $\phi$ is a measurable function $f: \Omega \rightarrow Y$ such that $f(\omega) \in \phi(\omega)$ for all $\omega \in \Omega$.

We now define the concept of a Caratheodory selection which combines the notion of continuous selection and measurable selection.

Let $(X, Q)$ be a measurable space and $Y$ and $Z$ be topological spaces. Let $\phi: X \times Z \rightarrow 2^{Y}$ be a (possibly empty-valued) correspondence. Let $U=\{(x, z) \in X \times Z: \phi(x, z) \neq \phi\}$. A Caratheodory selection for $\phi$ is a function $f: U \rightarrow Y$ such that $f(x, z) \in \phi(x, z)$ for $a l l(x, z) \in U$ and; for each $x \in X, f(x, \cdot)$ is continuous on $U^{X}=\{z \in Z:(x, z) \in U\}$ and for each $z \in Z, f(\cdot, z)$ is measurable on $U^{z}=\{x \in X:(x, z) \in U\}$.

If $(X, Q)$ and $(Y, B)$ are measurable spaces and $\phi: X \rightarrow 2^{Y}$ is a correspondence, $\phi$ is said to have a measurable graph if $G_{\phi}$ belongs to the product o-algebra $\alpha \otimes B$. We are usually interested in the situation where $(x, Q)$ is a measurable space, $Y$ is a topological space and $B=B(Y)$ is the Borel $\sigma$-algebra of $\mathrm{Y}$. For a correspondence $\phi$ from a measurable space into a topological space, if we say that $\phi$ has a measurable graph, it is understood that the 
topological space is endowed with its Borel o-algebra (unless specified otherwise). In the same setting as above $i . e .,(x, Q)$ a measurable space and $Y$ a topological space, $\phi$ is sald to be lower measurable if $\{\mathrm{x}: \phi(\mathrm{x}) \cap \mathrm{V} \neq \phi\} \in \boldsymbol{Q}$ for every $\mathrm{V}$ open in $\mathrm{Y}$.

The following facts will be useful in the sequel.

(F.2.3) Let $(\Omega, \boldsymbol{Q}, \mu)$ be a complete finite measure space $\mathrm{X}$ be a separable metric space and $\phi: \Omega \rightarrow 2^{\mathrm{X}}$ be a nonempty valued correspondence having a measurable graph, i.e., $G_{\phi} \in \boldsymbol{a} \otimes B(\mathrm{X})$. Then there exists a measurable selection for $\phi[5$, Theorem III.22, p. 22, or 6, Theorem 5.2, p. 60]

(F.2.4) Let $(\Omega, Q, \mu)$ be a complete finite measure space, $\mathrm{X}$ be a complete separable metric space and $\phi: \Omega \times x \rightarrow \mathbb{R}^{\ell}$ be a convex (possibly empty-) valued correspondence such that

(i) $\phi(\cdot, \cdot)$ is lower measurable with respect to the $\sigma$-algebra $\alpha \otimes B(x)$, and

(11) for each $\omega \in \Omega, \phi(\omega, \cdot)$ is 1.s.c.

Then there exists a Caratheodory selection for $\phi[12$, Theorem 3.2] .

(F.2.5) The previous fact remains true if $\phi$ is a correspondence from $\Omega \times X$ 1nto $2^{Y}$, where $Y$ is a separable Banach space and $(f)$ and $(i i)$ are replaced by

(i') $G_{\phi} \in a \otimes B(x) \otimes B(Y)$ and

(ii') for each $\omega \in \Omega, \phi(\omega, \cdot)$ has an open graph, i.e., for each $\omega \in \Omega$ the set $G_{\phi(\omega, \cdot)}=\{(x, y) \in X \times Y: y \in \phi(\omega, x)\}$ is open in $X \times Y$ [13, Main Theorem]. 
(F.2.6) Let $\Omega$ be a measurable space $\left\{Y_{i}: i \in I\right\}$, (where I is a countable set) be a family of second countable topological spaces. Let $Y=\prod_{i \in Y^{i}}$. If for each $i \in I, F_{i}: \Omega \rightarrow 2^{Y_{i}}$ is lower measurable then the correspondence $F: \Omega \rightarrow 2^{Y}$ defined by $F(\omega)=\underset{i \in I_{i}}{F_{i}}(\omega)$ is also lower measurable $[11$, Proposition 2.3, p. 55].

(F.2.7) Let $\Omega$ be a measurable space, $X$ be a separable metric space and for each $i \in I$, (where $I$ is a countable set) $F_{i}: \Omega \rightarrow 2^{X}$ is a lower measurable and closed valued correspondence. Suppose that for each $\omega \in \Omega$, $F_{i}(\omega)$ is compact for at least one $i \in I$. Then the correspondence $F: \Omega \rightarrow 2^{X}$ defined by $F(\omega)=\bigcap_{i \in I} F_{i}(\omega)$ is lower measurable $[11$, Theorem $4.1, p .58]$.

If $(X, Q),(Y, B)$ and $(Z, \mathcal{Z})$ are measurable spaces, $U \subseteq X \times Z$ and $f: U \rightarrow Y$, we call $f$ jointly measurable if for every $B \in B, f^{-1}(B)=U \cap A$ for some $A \in a \otimes\}$. It is a standard result that if $z$ is a separable metric space, $Y$ is metric and $f: X \times Z+Y$ is such that for each fixed $x \in X, f(x, \cdot)$ is continuous and for each fixed $z \in Z, f(\cdot, z)$ is measurable, then $f$ is jointly measurable (where $B=B(y), z=\beta(Z)$ ). It turns out, that in several instances $U$ is a proper subset of $x \times z$, and this situation is more delicate. However, in this more delicate situation it can be shown that $\mathrm{f}$ is still jointly measurable. In particular, we have the following fact.

(F.2.8) Let $(\Omega, Q)$ be a measurable space, $\mathrm{x}$ be a separable metric space, $\mathrm{Y}$ be a metric space and $U \subseteq \Omega \times \mathrm{X}$ be such that

(i) for each $\omega \in \Omega$ the set $U^{\omega}=\{x \in X:(\omega, x) \in U\}$ is open in $X$, and (1i) for each $x \in x$ the set $U^{x}=\{\omega \in \Omega:(\omega, x) \in U\}$ belongs to $\boldsymbol{Q}$. 
Let $f: U \rightarrow Y$ be a function such that for each $\omega \in \Omega, f(\omega, \cdot)$ is continuous on $U^{\omega}$ and for each $x \in X, f(\cdot, x)$ is measurable on $U^{x}$. Then $f$ is jointly relatively measurable with respect to the $\sigma$-algebra $\boldsymbol{a} \otimes \boldsymbol{B}(\mathrm{x})$, i.e. for every open subset $V$ of $Y,\{(\omega, x) \in U: f(\omega, x) \in V\}=U \cap A$ for some $\mathrm{A} \in \boldsymbol{a} \otimes B(\mathrm{x})[12$, Lemma 4.12].

(F.2.9) Let $(\Omega, \boldsymbol{Q}, \mu)$ be a complete measure space and $X$ be a complete separable metric space. If 0 belongs to $a \otimes B(x)$ its projection $\operatorname{proj}_{\Omega}(0)$ belongs to $\boldsymbol{Q},[5$, Theorem III.23, p. 75].

\subsection{The Random Coincidence Theorem}

The result below is a random version of Fan's Coincidence Theorem, [8, Theorem 6, p. 238].

Theorem 2.4.1: Let $\mathrm{X}$ be a compact convex nonempty subset of a locally convex separable and metrizable linear topological space $Y$ and let $(\Omega, \mathcal{F}, v)$ be a complete finite measure space. Let $\gamma: \Omega \times \mathrm{X} \rightarrow 2^{\mathrm{Y}}$ and $\mu: \Omega \times \mathrm{X} \rightarrow 2^{\mathrm{Y}}$ be two nonempty, convex, closed and at least one of them is compact valued correspondences such that:

(i) $\mu(\cdot, \cdot)$ and $\gamma(\cdot, \cdot)$ are lower measurable,

(ii) for each fixed $\omega \in \Omega$, the correspondences $\mu(\omega, \cdot): X \rightarrow 2^{Y}$ and $\gamma(\omega, \cdot): X \rightarrow 2^{Y}$ are u.s.c.

(11i) for every $\omega \in \Omega$ and every $x \in x$, there exist three points $y \in x$, $u \in \gamma(\omega, x), z \in \mu(\omega, x)$ and a real number $\lambda>0$ such that $y-x=$ $\lambda(u-z)$.

Then there exists a measurable function $x^{*}: \Omega \rightarrow \mathrm{X}$ such that $\gamma\left(\omega, x^{*}(\omega)\right) \cap \mu\left(\omega, x^{*}(\omega)\right) \neq \phi$ for almost all $\omega \in \Omega$. 
Proof: Define the correspondence $W: \Omega \times X \rightarrow 2^{Y}$ by $W(\omega, x)=$ $\gamma(\omega, \mathbf{x}) \cap \mu(\omega, \mathbf{x})$. Since $\gamma(\cdot, \cdot)$ and $\mu(\cdot, \cdot)$ are closed valued and lower measurable and at least one of them is compact valued, it follows from (F.2.7) that $W(\cdot, \cdot)$ is lower measurable. Define the correspondence $\phi: \Omega \rightarrow 2^{X}$ by

$$
\phi(\omega)=\{x \in X: W(\omega, x) \neq \phi\} .
$$

Observe that

$$
\begin{aligned}
G_{\phi}=\{(\omega, x) \in \Omega \times x: x \in \phi(\omega)\} & =\{(\omega, x) \in \Omega \times x: W(\omega, x) \neq \phi\} \\
& =\{(\omega, x) \in \Omega \times x: W(\omega, x) \cap Y \neq \phi\},
\end{aligned}
$$

and the latter set belongs to $\mathcal{B} \otimes(\mathrm{X})$ since $\mathrm{W}(\cdot, \cdot)$ is lower measurable.

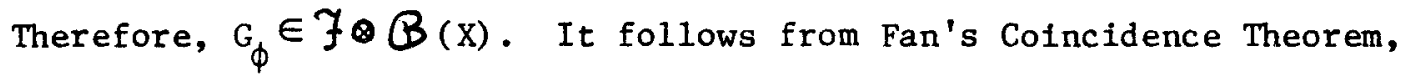
(Fan $[8$ Theorem 6, p. 238]) that for each $\omega \in \Omega, \phi(\omega) \neq \phi$. Thus, the correspondence $\phi: \Omega \rightarrow 2^{X}$ satisfies all the conditions of (F.2.3), (the Aumann Measurable Selection Theorem) and consequently, there exists a measurable function $x^{*}: \Omega \rightarrow X$ such that $x^{*}(\omega) \in_{\phi}(\omega)$ for almost all $\omega$ in $\Omega$, i.e., $\gamma\left(\omega, x^{*}(\omega)\right) \cap \mu\left(\omega, x^{*}(\omega)\right) \neq \phi$ for almost all $\omega$ in $\Omega$. This completes the proof of the Theorem.

An immediate corollary of the above theorem is a random version of the Kakutani-Fan fixed point theorem (see [7, Theorem 1, p. 122]).

Corollary 2.4.1: Let $\mathrm{X}$ be a compact, convex, non-empty subset of a locally convex separable and metrizable linear topological space $Y$ and let $(\Omega, \mathcal{F}, v)$ be a complete finite measure space. Let $\gamma: \Omega \times x \rightarrow 2^{\mathrm{X}}$ be a nonempty, convex closed valued correspondence such that for each fixed $\omega \in \Omega, \gamma(\omega, \cdot)$ is u.s.c.. and $\gamma(\cdot, \cdot)$ is lower measurable. Then $\gamma(\cdot, \cdot)$ has a random flxed point, $1 . e .$, there exists a measurable function $x^{*}: \Omega+x$ such that $x *(\omega) \in \gamma(\omega, x *(\omega))$ for almost all $\omega$ in $\Omega$. 
Proof: Define the correspondence $\mu: \Omega \times X \rightarrow 2^{X}$ by $\mu(\omega, x)=\{x\}$. Clearly for each fixed $\omega \in \Omega, \mu(\omega, \cdot)$ is u.s.c. and $\mu(\cdot, \cdot)$ is convex, lower measurable, nonempty, compact valued. Let $x \in X$ and $\omega \in \Omega$. By choosing $u \in \gamma(\omega, x), \quad z=x \in \mu(\omega, x)$ and $\lambda \in(0,1)$ assumption (iif) of Theorem 2.4.1 is satisfied (simply notice that since $\mathrm{X}$ is convex $y=x+\lambda(u-z)=\lambda u+(1-\lambda) x \in x)$. Hence, by the previous theorem there exists a measurable function $x^{*}: \Omega \rightarrow x$ such that $\gamma\left(\omega, x^{*}(\omega)\right) \cap \mu\left(\omega, x^{\star}(\omega)\right) \neq \phi$ for almost all $\omega \in \Omega$, 1.e., $x^{*}(\omega) \in \gamma\left(\omega, x^{*}(\omega)\right)$ for almost all $\omega \in \Omega$. 


\section{THE MAIN THEOREMS}

\subsection{Random Games and Random Equilibria}

Let $(\Omega, \mathcal{F}, \mu)$ be a complete finite measure space. We interpret $\Omega$ as the states of nature of the world and assume that $\Omega$ is large enough to include all the events that we consider to be interesting. I will denote the $\sigma$-field of events. Denote by $I$ the set of players. I can be any finite or countably infinite set. A random game

$E=\left\{\left(X_{i}, P_{i}\right): i \in I\right\}$ is a set of ordered pairs $\left(X_{i}, P_{i}\right)$ where

(1) $x_{i}$ is the strategy set of player 1 , and

(2) $P_{i}: \Omega \times X \rightarrow 2^{X_{i}}$ (where $X=\prod_{i \in I_{1}} X_{1}$ is the preference (or choice) correspondence of player $i$.

We read $y_{i} \in P_{i}(\omega, x)$ as player $i$ strictly prefers $y_{i}$ to $x_{i}$ at the state of nature $\omega$, if the (given) components of other players are fixed.

A random equilibrium for $\mathrm{E}$ is a measurable function $\mathrm{x}^{*}: \Omega \rightarrow \mathrm{X}$ such that for all $i \in I, P_{i}\left(\omega, x^{*}(\omega)\right)=\phi$ for almost all $\omega \in \Omega$.

Notice that each player in the game described above is characterized by a strategy set and a preference correspondence. We now follow the original formulation by Nash [18] (and his generalizations by Fan $[6]$ and Browder [3] among others) where preference correspondences are replaced by payoff functions, i.e., real valued functions defined on $\Omega \times \mathrm{x}$.

Let $\Gamma=\left\{\left(\mathrm{X}_{i}, u_{i}\right): i \in I\right\}$ be a Nash-type random game, $1 . e .$,

(i) $X_{1}$ is the strategy set of player $i$, and

(i1) $u_{1}: \Omega \times X \rightarrow \mathbb{R}$, (where $X=\prod_{i \in I} x_{i}$ ) is the payoff function of player 1 . 
Let $\tilde{x}_{i}=\prod_{j \neq i} x_{j}$ and denote the points of $\tilde{x}_{i}$ by $\tilde{x}_{i}$.

A random Nash equilibrium for $\Gamma$ is a measurable function $x *: \Omega \rightarrow \mathrm{X}$ such that for all $i$,

$u_{i}\left(\omega, x^{*}(\omega)\right)=\max _{y_{i} \in x_{i}} u_{i}\left(\omega, y_{i} x_{i}^{*}(\omega)\right)$ for almost all $\omega \in \Omega$.

We now state our first random equilibrium existence result.

Theorem 3.1: Let $E=\left\{\left(X_{i}, P_{i}\right): i \in I\right\}$ be a random game satisfying for each $i$ the following assumptions:

(a.1.1) $x_{i}$ is a compact, convex, nonempty subset of $\mathbb{R}^{l}$,

(a.1.2) $\operatorname{conP}_{i}(\cdot, \cdot)$ is lower measurable, i.e., for every open subset $V$ of $x_{i}$, the set $\left\{(\omega, x) \in \Omega \times x: \operatorname{conP}_{i}(\omega, x) \cap V \neq \phi\right\}$ belongs to $7 \otimes B(x)$,

(a.1.3) for every measurable function $x: \Omega \rightarrow x, x_{i}(\omega) \notin \operatorname{con} P_{i}(\omega, x(\omega))$ for almost all $\omega \in \Omega$,

(a.1.4) for each fixed $\omega \in \Omega, P_{i}(\omega, \cdot)$ is 1.s.c.

Then there exists a random equilibrium for $E$.

As a Corollary of Theorem 3.1 we obtain a generalized random version of Nash's [ 18 Theorem 1, p. 288] equilibrium existence result.

Corollary 3.1: Let $\Gamma=\left\{\left(\mathrm{X}_{i}, \mathrm{u}_{i}\right): i \in I\right\}$ be a Nash-type random game satisfying for each $i$ the following assumptions :

(c.1.1) $x_{i}$ is a compact, convex, nonempty subset of $\mathbb{R}^{\ell}$, 


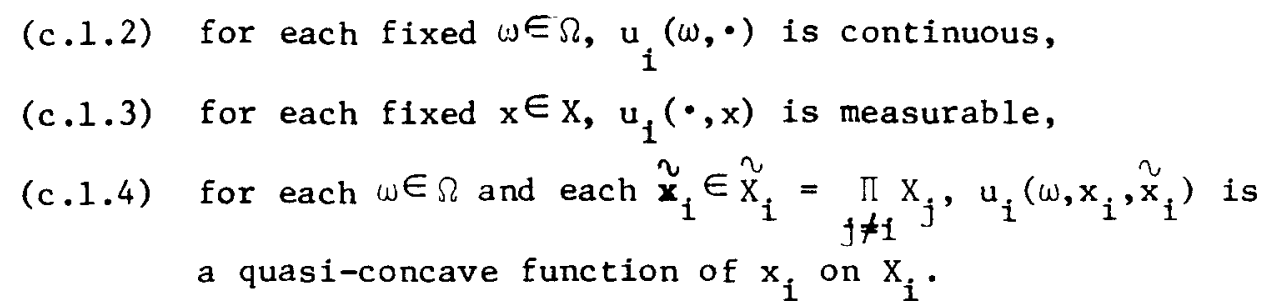

Then there exists a random Nash equilibrium for $\Gamma$.

Theorem 3.2: Let $E=\left\{\left(X_{i}, P_{i}\right): i \in I\right\}$ be a random game satisfying for each $i$ the following assumptions :

(a.2.1) $x_{i}$ is a compact, convex, nonempty subset of a separable Banach space,

(a.2.2) $\operatorname{conP}_{i}(\cdot, \cdot)$ has a measurable graph, i.e., the set $\left\{\left(\omega, x, y_{i}\right) \in \Omega \times x \times x_{i}: y_{i} \in \operatorname{conP}_{i}(\omega, x)\right\} \in \mathcal{B} \otimes(x) \otimes B\left(x_{i}\right)$,

(a.2.3) for every measurable function $x: \Omega \rightarrow x$, $\mathrm{x}_{i}(\omega) \notin \operatorname{conP}_{i}(\omega, \mathrm{x}(\omega))$ for almost all $\omega \in \Omega$,

(a.2.4) for each $\omega \in \Omega, P_{i}(\omega, \cdot)$ has an open graph in $x \times x_{i}$.

Then $E$ has a random equilibrium.

The following Corollary of Theorem 3.2 extends Corollary 3.1 to strategy sets which can be subsets of arbitrary separable Banach spaces. We thus have a random version of Nash's result [18, Theorem 1, p. 288] in separable Banach spaces. It should be noted that Corollary 3.2 may be seen as a random generalization of the deterministic equilibrium existence results of Fan [9, Theorem 4, p. 192] and Browder [4, Theorem 14, p. 277], but only if the underlying strategy space is separable. It is worth noting that Fan and Browder allow only for a finite number of players whereas in our setting the set of players can be any finite or countably infinite set. 
Corollary 3.2: Replace assumption (c.1.1) in Corollary 3.1 by

$$
\begin{aligned}
& \text { (c.1.1') } x_{i} \text { is a nonempty, compact, convex subset of a separable } \\
& \text { Banach space. }
\end{aligned}
$$

Then the conclusion of Corollary 3.1 remains true.

A couple of comments are in order. Notice that the continuity assumption in Theorem 3.1, i.e., (a.1.4) is weaker than the continuity assumption $(a .2 .4)$ of Theorem 3.2. The reason we need a weaker continuity assumption is that the proof of Theorem 3.1 makes use of (F.2.4) which is a Caratheodory selection result for a correspondence which is lower measurable in one variable and 1.s.c. in the other. However, in the proof of Theorem 3.2 a different Caratheodory selection result is used, i.e., (F.2.5), which requires a stronger continuity assumption. Moreover, observe that Corollary 3.1 follows directly from Corollary 3.2. Nevertheless, we choose to state Corollary 3.1 since its proof by means of Theorem 3.1 is slightly different than the proof of Corollary 3.2 which follows from Theorem 3.2. Finally it is important to note that the proofs of Theorems 3.1 and 3.2 do not use any deterministic equilibrium existence results. To the contrary, our arguments "start from scratch" and provide alternative ways to prove the equilibrium results of Nash, Fan and Browder. 3 We now turn to the problem of the existence of equilibrium points for Bayesian games with a countable number of players.

\subsection{Bayesian Games and Bayestan Equilibria}

Let $(\Omega, \mathcal{F}, \mu)$ be a complete fintte measure space as described in Section 3.1. We still denote by $I$ the set of players where $I$ can be any 
finite or countably infinite set. A Bayesian game $G=\left\{\left(X_{i}, h_{i}, S_{i}, q\right)\right.$ : $i \in I\}$ is a set of quadruples $\left(x_{i}, h_{i}, s_{i}, q_{i}\right)$ where,

(1) $x_{1}$ is the strategy set of player 1 .

(2) $h_{i}: \Omega \times X \rightarrow \mathbb{R}$ (where $X=\prod_{i \in I} X_{i}$ ) is the payoff function of player $i$,

(3) $s_{i}$ is a measurable partition of $(\Omega, \mathcal{F}, \mu)$ denoting the information available to player 1 ,

(4) $q_{i}: \Omega \rightarrow \mathbb{R}_{++}$(where $\mathbb{R}_{++}$denotes the strictly positive elements of $\mathbb{R}$ ), is the prior of player $i$.

As in Aumann [2] or Myerson [1]] it is assumed that $G=\left\{\left(x_{i}, h_{i}, s_{i}, q_{i}\right)\right.$ : $i \in I\}$, is common knowledge, 1.e., every player knows $G$, every player knows that every player knows $G$, every player knows that every player knows that every player knows $G$ and so on. For each $i \in I$, denote by $E_{i}(\omega)$ the event in $S_{i}$ which contains $\omega \in \Omega$. Given $E_{i}(\omega)$ in $S_{i}$ the conditional expected utility of player $1, v_{1}: \Omega \times \mathrm{X} \rightarrow \mathbb{R}$ is defined by $(3.1) v_{i}(\omega, x)=\int_{t \in E_{1}(\omega)} q_{1}\left(t \mid E_{i}(\omega)\right) h_{i}(t, x) d \mu(t)$
where

$$
q_{i}\left(t \mid E_{i}(\omega)\right) d \mu(t)= \begin{cases}0 & \text { if } t \notin E_{i}(\omega) \\ \frac{q_{i}(t) d \mu(t)}{\int q_{i}(s) d \mu(s)} & \text { if } t \in E_{i}(\omega) .\end{cases}
$$

A Bayesian equilibrium for $G=\left\{\left(x_{i}, h_{i}, S_{i}, q_{i}\right): i \in I\right\}$ is a function $x^{*}: \Omega \rightarrow X$ such that, each $x^{*}(\cdot)$ is $S_{i}$-measurable and for each $i \in I$ $v_{i}\left(\omega, x^{*}(\omega)\right)=\max _{y_{i} \in x_{i}} v_{i}\left(\omega, y_{i}, \tilde{x}_{i}^{*}(\omega)\right)$ for almost all $\omega \in \Omega$ 
(where $v_{i}$ is given by (3.1)).

We are now ready to state our Bayesian equilibrium existence theorem.

Theorem 3.3: Let $G=\left\{\left(\mathrm{X}_{1}, \mathrm{~h}_{1}, \mathrm{~S}_{1}, \mathrm{q}_{1}\right): i \in I\right\}$ be a Bayesian game satisfying for each $i \in I$ the following assumptions:

(a.3.1) $x_{1}$ is a compact, convex, nonempty subset of a Euclidean space $\mathbb{R}^{n}$,

(a.3.2) for each fixed $\omega \in \Omega, h_{i}(\omega, \cdot)$ is continuous,

(a.3.3) for each fixed $x \in X, h_{i}(\cdot, x)$ is measurable,

(a.3.4) for each $\omega \in \Omega$ and each $\tilde{x}_{1} \in \tilde{x}_{i}=\prod_{j \neq 1} x_{j}$,

$h_{i}\left(\omega, x_{1}, x_{i}\right)$ is a quasi-concave function of $x_{i}$ on $x_{i}$,

(a.3.5) $h_{i}$ is integrably bounded, 4

(a.3.6) $q_{1}(\cdot)$ is measurable.

Then $G$ has a Bayesian equilibrium.

Before we turn to the proof of our theorems we would like to note that all the equilibrium existence results of Section 3 can be easily extended to abstract economies as defined in [6], [24], [25]. Moreover, one can use the equilibrium results for abstract economies to obtain equilibrium existence theorems for random exchange economies or Bayesian exchange economies. In particular, in this setting of incomplete information the appropriate equilibrium notion is that of a rational expectations equilibrium. We will take up these deta1ls, however, in a subsequent paper. 
4. PROOF OF THE MAIN THEOREMS

\subsection{Lemata}

We begin by proving two Lemmata which are going to be needed in the seque1.

Lemma 4.1: Let $(S, Q, \mu)$ be a complete measure space and $X, Y$ be separable metric spaces. Let $\phi: \mathrm{S} \times \mathrm{X} \rightarrow 2^{\mathrm{Y}}$ be a lower measurable (possibly empty-valued) correspondence. Suppose that for each fixed $s \in s, \phi(s, \cdot)$ is 1.s.c. Let $O=\{(s, x) \in \mathrm{S} \times \mathrm{X}: \phi(\mathrm{s}, \mathrm{x}) \neq \phi\}$, and let $\mathrm{f}: 0 \rightarrow \mathrm{Y}$ be a Caratheodory selection for $\phi$. Then the correspondence $\theta: S \times X \rightarrow 2^{Y}$ defined by

$$
\theta(s, x)= \begin{cases}\{f(s, x)\} & \text { if }(s, x) \in_{0} \\ Y & \text { if }(s, x) \in_{0}\end{cases}
$$

is lower measurable.

Proof: We begin by making a couple of observations. First notice that, since $\phi(\cdot, \cdot)$ is lower measurable the set $0=\{(s, x) \in s \times x: \phi(s, x) \neq \phi\}=$ $\{(s, x) \in s \times x: \phi(s, x) \cap y \neq \phi\}$ belongs to $\boldsymbol{a} \otimes B(X)$. By (F.2.9) for each $x \in x$ the set

$$
\begin{aligned}
0^{x}=\{s \in s:(s, x) \in 0\} & =\operatorname{proj}_{S}(\{(s, x) \in s \times x: \phi(s, x) \neq \phi\} \cap(s \times\{x\})) \\
& =\operatorname{proj}_{S}(0 \cap(s \times\{x\})),
\end{aligned}
$$

belongs to $\boldsymbol{\alpha}$. Moreover, notice that since for each fixed $s \in s, \phi(s, \cdot)$

is 1.s.c. It follows that for each $s \in S$ the set $0^{S}=\{x \in X$ :

$(s, x) \in 0\}$ is open in $x$. Since for each fixed $s \in s, f(s, \cdot)$ is con-

tinuous on $o^{s}$ and for each fixed $x \in s, f(\cdot, x)$ is measurable on $0^{x}$, by

(F.2.8) $\mathrm{f}(\cdot, \cdot)$ is fointly measurable. It can be easily now seen that for

every open subset $V$ of $Y$ the set $A=\{(s, x) \in S \times X: \theta(s, x) \cap V \neq \phi\}=B \cup C$ 
where $B=\{(s, x) \in 0: f(s, x) \in V\}$ and $C=\{(s, x) \in S \times X \backslash 0: Y \cap V \neq \phi\}$. clearly, $\mathrm{B} \in a_{\otimes} \otimes(\mathrm{x})$ and $\mathrm{c} \in \alpha_{\otimes} \otimes(\mathrm{x})$ and therefore $\mathrm{A}=\mathrm{B} \cup_{C}$ belongs to $a \otimes B(x)$. Consequently, $\theta(\cdot, \cdot)$ is lower measurable as was to be shown.

Lemma 4.2: Let $(s, a)$ be a measurable space, $Z$ be a separable metric space and $\widetilde{\mathbb{R}}$ be the extended real line. Let $g: S \times Z \rightarrow \widetilde{R}$ be a function such that for each fixed $s \in S, g(s, \cdot)$ is continuous and for each fixed $x \in z, g(\cdot, z)$ is measurable. Define the correspondence $\mathrm{K}: \mathrm{S} \rightarrow 2^{\mathrm{Z}}$ by

$$
K(s)=\{z \in Z: g(s, z)>0\} .
$$

Then, (a) $G_{K} \in a \otimes B(z)$, i.e., $K(\cdot)$ has a measurable graph, and (b) $K(\cdot)$ is lower measurable.

Proof: (a) Since for each fixed $s \in s, g(s, \cdot)$ is continuous and for each fixed $z \in z, g(\cdot, z)$ is jointly measurable, it follows from a standard result that $g(\cdot, \cdot)$ is jointly measurable. Observe that,

$$
\begin{aligned}
g^{-1}((0,+\infty)) & =\{(s, z) \in S \times z: g(s, z)>0\} \\
& =\{(s, z) \in s \times z: z \in K(s)\} \\
& =G_{K},
\end{aligned}
$$

and the latter set belongs to $a \otimes B(z)$ since $g(\cdot, \cdot)$ is jointly measurable.

(b) We must show that the set $\{s \in s: K(s) \cap v \neq \phi$ \} belongs to $a$ for every open subset $V$ of $z$. As it was remarked above, $g(\cdot, \cdot)$ is jointly measurable, $1 . e ., g$ is measurable with respect to the product $\sigma$-algebra $a \otimes B(z)$. Let $D$ be a countable dense subset of $z$, and let $U=(0,+\infty)$. Observe that, 


$$
\begin{aligned}
\{s: \mathrm{K}(s) \cap \mathrm{V} \neq \phi\} & =\{s: g(s, z) \in U \text { for some } z \in V\} \\
& =\{s: g(s, d) \in U \text { for some } d \in D\} \\
& =\underset{d \in D}{U}\{s: g(s, d) \in U\}
\end{aligned}
$$

and the latter set belongs to $\boldsymbol{Q}$ since for each fixed $z \in z, g(\cdot, z)$ is measurable. This completes the proof of the Lemma.

\subsection{Proof of Theorem 3.1}

For each $1 \in I$ define the correspondence $\phi_{1}: \Omega \times x+2^{X_{1}}$ by $\phi_{i}(\omega, x)=$ $\operatorname{conP} P_{i}(\omega, x)$. Since by assumption $(a .1 .4)$ for each fixed $\omega \in \Omega, P_{i}(\omega, \cdot)$ is 1.s.c. it follows from (F.2.1) that for each fixed $\omega \in \Omega, \phi_{i}(\omega, \cdot)$ is $1 . s . c$. Furthermore, by assumption $(a .1 .2), \phi_{i}(\cdot, \cdot)$ is lower measurable and clearly convex valued. For $i \in I$ let $0_{i}=\left\{(\omega, x) \in \Omega \times x: \phi_{i}(\omega, x) \neq \phi\right\}$. For each $\omega \in \Omega$ let $0_{i}^{\omega}=\left\{x \in X:(\omega, x) \in 0_{i}\right\}$ and for each $x \in X$ let $0_{i}^{x}=\{\omega \in \Omega$ : $\left.(\omega, x) \in 0_{1}\right\}$. It follows from (F. 2.4) that there exists a Caratheodory selection for $\phi_{i}$, i.e., there exists a function $f_{i}: o_{i} \rightarrow x_{i}$ such that $f_{i}(\omega, x) \in \phi_{i}(\omega, x)$ for all $(\omega, x) \in o_{i}$ and for each $x \in x, f_{i}(\cdot, x)$ is measurable on $o_{i}^{x}$ and for each $\omega \in \Omega, f_{i}(\omega, \cdot)$ is continuous on $o_{i}^{\omega}$. For each $i \in I$ define the correspondence $F_{i}: \Omega \times x \rightarrow 2^{X_{i}}$ by

$$
F_{i}(\omega, x)= \begin{cases}\left\{f_{1}(\omega, x)\right\} & \text { if }(\omega, x) \in o_{1} \\ x_{i} & \text { if }(\omega, x) \notin o_{i} .\end{cases}
$$

By Lemma $4.1, \mathrm{~F}_{i}(\cdot, \cdot)$ is lower measurable, and it is obviously closed, convex, nonempty valued. Since for each fixed $\omega \in \Omega, \phi_{i}(\omega, \cdot)$ is 1.s.c. the set $o_{i}^{\omega}=\left\{x \in X:(\omega, x) \in o_{i}\right\}=\left\{x \in X: \phi_{i}(\omega, x) \neq \phi\right\}=\left\{x \in x: \phi_{i}(\omega, x) \cap x_{i} \neq \phi\right\}$ 
is open in the relative topology of $x$, and consequently for each fixed $\omega \in \Omega$, $F_{i}(\omega, \cdot)$ is u.s.c. Define the correspondence $F: \Omega \times x \rightarrow 2^{X}$ by $F(\omega, x)=$ $\operatorname{II}_{i \in I_{i}}(\omega, x)$. Since for each $1, F_{i}(\cdot, \cdot)$ is lower measurable it follows from $(F .2 .6)$ that $\mathrm{F}(\cdot, \cdot)$ is lower measurable as well. Obviously $\mathrm{F}(\cdot, \cdot)$ is closed, convex and nonempty valued. By $(F .2 .2)$ for each fixed $\omega \in \Omega, F(\omega, \cdot): X \rightarrow 2^{X}$ is u.s.c. Therefore, $F(\cdot, \cdot)$ satisfies all the conditions of Corollary 2.4.1 and consequently there exists a random fixed point, i.e., there exists a measurable function $x^{*}: \Omega \rightarrow \mathrm{X}$ such that $\mathrm{x}^{\star}(\omega) \in \mathrm{F}\left(\omega, \mathrm{x}^{*}(\omega)\right)$ for almost a1l $\omega \in \Omega$. We now show that the random fixed point is by construction a random equilibrium for the game $E$. Notice that for each $i \in I$, if $\left(\omega, x^{*}(\omega)\right) \in 0_{i}$, then by the definition of $F_{1}, x_{1}^{*}(\omega)=f_{i}\left(\omega, x^{*}(\omega)\right) \in \operatorname{conP}_{i}\left(\omega, x^{*}(\omega)\right)$, a contradiction to assumption (a.1.3). Thus, for all $i \in I,\left(\omega, x^{*}(\omega)\right) \otimes_{0}$ for almost all $\omega \in \Omega$, i.e., for all $i \in I, \operatorname{conP}_{1}\left(\omega, x^{*}(\omega)\right)=\phi$ for almost all $\omega \in \Omega$ which in turn implies that for all $i \in I, P_{i}\left(\omega, x^{*}(\omega)\right)=\phi$ for almost all $\omega \in \Omega$, i.e., $\mathrm{x}^{*}: \Omega \rightarrow \mathrm{X}$ is a random equilibrium for $\mathrm{E}$. This completes the proof of the Theorem.

\subsection{Proof of Corollary 3.1}

For each $i \in I$, define the correspondence $Q_{i}: \Omega \times x \rightarrow 2^{X_{i}}$ by $Q_{i}(\omega, x)=\left\{y_{i} \in x_{i}: h_{i}\left(\omega, x, y_{i}\right)>0\right\}$, where $h_{i}\left(\omega, x, y_{i}\right)=u_{i}\left(\omega, y_{i}, \tilde{x}_{i}\right)-u_{i}(\omega, x)$. Setting $s=\Omega \times x, z=x_{i}, \quad \boldsymbol{\alpha}=\boldsymbol{F} \otimes Q(\mathrm{x}), g(s, z)=h_{i}\left(\omega, x, y_{i}\right), k(s)=Q_{i}(\omega, x)$ for $s=(\omega, x)$ in Lemma $4.2(b)$ we can conclude that $Q_{1}(\cdot, \cdot)$ is lower measurable. It follows from assumption (c.1.4) that, $Q_{i}(\cdot, \cdot)$ is convex valued, and clearly for any measurable function $x: \Omega \rightarrow x, x_{i}(\omega) \notin \operatorname{con} Q_{i}(\omega, x(\omega))=$ $Q_{1}(\omega, x(\omega))$ for almost all $\omega \in \Omega$. Moreover, it follows from assumption (c.1.2) that for each fixed $\omega \in \Omega, \quad Q_{1}(\omega, \cdot)$ has an open graph in $X \times X_{i} \cdot$ Hence, the random game $E=\left\{\left(X_{1}, Q_{1}\right): i \in I\right\}$ satisfies all the assumptions of Theorem 3.1 and therefore $\mathrm{E}$ has a random equilibrium, 1.e., there exists a measurable function $x^{*}: \Omega \rightarrow \mathrm{X}$ such that 
for all $i, Q_{i}\left(\omega, x^{*}(\omega)\right)=\phi$ for almost all $\omega \in \Omega$. But this implies that for all i, $u_{i}\left(\omega, x^{*}(\omega)\right)=\max _{y_{1} \in x_{1}} u_{i}\left(\omega, y_{1}, x_{i}^{*}(\omega)\right)$, for almost all $\omega \in \Omega$, i.e., $x^{*}: \Omega \rightarrow x$ is a random Nash equitlibrium for the game $\Gamma=\left\{\left(x_{i}, u_{i}\right): i \in I\right\}$. This completes the proof of the Corollary.

\subsection{Proof of Theorem 3.2}

For each $i \in I$ define the correspondence $\phi_{i}: \Omega \times x+2^{X_{1}}$ by $\phi_{1}(\omega, x)=$ $\operatorname{conP}_{i}(\omega, x)$. Since by assumption $(a .2 .4)$ for each $\omega \in \Omega, P_{i}(\omega, \cdot)$ has an open graph in $x \times x_{1}$ it can be easily checked that so does $\phi_{1}(\omega, \cdot)$ for each $\omega \in \Omega$.

Let $o_{1}=\left\{(\omega, x) \in \Omega \times x: \phi_{1}(\omega, x) \neq \phi\right\}$. Since $\phi_{i}(\cdot, \cdot)$ has a measurable graph (recall assumption $(a .2 .2)$ ) and it is convex valued appealing to (F.2.5) we can ensure the existence of a Caratheodory selection for $\phi_{i}$. One can now proceed as in the proof of Theorem 3.1 to complete the proof.

\subsection{Proof of Corollary 3.2}

The proof is identical with that of Corollary 3.1 except with the fact that one now has to use Lemma $4.2(a)$ to show that $Q_{i}(\cdot, \cdot)$ has a measurable graph, and appeal to Theorem 3.2 instead of Theorem 3.1.

\subsection{Proof of Theorem 3.3}

The result follows directly from Corollary 3.1 . To see this note that since for each fixed $\omega \in \Omega, h_{i}(\omega, \cdot)$ is continuous and $h_{i}$ is integrably bounded by virtue of the Lebesgue dominated convergence theorem we can automatically conclude that for each fixed $\omega \in \Omega$, $v_{i}(\omega, \cdot)=\int_{t \in E_{i}(\omega)} q_{i}\left(t \mid E_{i}(\omega)\right) h_{i}(t, \cdot) d \mu(t)$ is sontinuous, (where 


$$
q_{1}\left(t \mid E_{i}(\omega)\right)=0 \text { if } t \in E_{i}(\omega) \text { and } q_{1}\left(t \mid E_{1}(\omega)\right) d \mu(t)=\frac{q_{i}(t) d \mu(t)}{\int q_{i}(s) d \mu(s)}
$$

if $\left.t \in E_{i}(\omega)\right)$.

Furthermore, it can be easily seen that for

each fixed $x \in X, v_{i}(\cdot, x)$ is $s_{i}$-measurable. Finally, it follows from assumption (a.3.4) that for each $\omega \in \Omega$ and each $\tilde{x}_{1} \in \tilde{x}_{i}={ }_{j \neq i} x_{j}, v_{i}\left(\omega, x_{i}, \tilde{x}_{i}\right)$ is a quasi-concave function of $x_{1}$ on $x_{1}$. We may now consider the Bayesian game $G=\left\{\left(X_{i}, h_{i}, S_{i}, q_{i}\right): i \in I\right\}$ as a random game $E=\left\{\left(X_{i}, v_{i}\right): i \in I\right\}$. Obviously the existence of a random Nash equilibrium for $E$ implies the existence of a Bayesian equilibrium for the game $G$. It can be easily seen that the random game E, satisfles all the assumptions of Corollary 3.1, and consequently, $E$ has a random Nash equilibrium. ${ }^{5}$ Hence, there exists a function $x *: \Omega \rightarrow X$ such that $x{ }_{i}(\cdot)$ is $S_{1}$-measurable and for all $i \in I$ $v_{i}(\omega, x *(\omega))=\max _{y_{1} \in x_{1}} v_{i}\left(\omega, y_{1}, n_{1}^{*}(\omega)\right)$ for almost all $\omega \in \Omega, i . e ., x^{*}(\cdot)$ is a Bayestan equilibrium for the game G. This completes the proof of the theorem. 


\section{RELATED LITERATURE}

The equilibrium existence results for games with incomplete information which are related to our Theorem 3.3 and we are aware of, are those in Balder [3], Milgrom-Weber [16] and Radner-Rosenthal [23]. 6

Their approach is based on distributional strategies and it is entirely different than ours, which is based on measurable functions. For purposes of comparison it may be instructive to briefly outline their approach.

Following [16] a game $G$ is a sextuple $\left\langle N,\left\{T_{1}\right\}_{i \in N},\left\{A_{1}\right\}_{i \in N}, \zeta\right\rangle$ where,

(1) $N=\{1,2, \ldots, n\}$ is the set of players.

(2) $\left\{T_{1}: i \in N\right\}$ is the set of types for each player. Each $\mathrm{T}_{1}$ is a complete, separable metric space.

(3) $\left\{A_{i}: i \in N\right\}$ is the set of actions for each player. Each $A_{i}$ is a compact metric space.

(4) $T_{0}$ is the set of possible states. $T_{0}$ is a complete, separable metric space.

(5) $u_{i}: T \times A+\mathbb{R}$, (where $T=T_{0} \times \ldots \times T_{n}$, $\left.A=A_{1} \times \ldots \times A_{n}\right)$ is the payoff function of player 1 . Each $u_{1}$ is bounded and measurable.

(6) $\zeta$ is the Information structure, where $\zeta$ is a probability measure on the Borel subsets of $\mathrm{T}$. Denote by $\zeta_{1}$ the marginal distribution on each $T_{1}$.

A distributional strategy for player 1 is a probability measure $\mu_{1}$ on the Borel subsets of $T_{1} \times A_{1}$, for which the marginal distribution of $T_{1}$ is $\zeta_{i}$. The expected payoff of player 1 is: 
(4.1) $v_{i}\left(\mu_{1}, \ldots, \mu_{n}\right)=\int u_{1}(t, a) \mu_{1}\left(d a_{1} \mid t_{1}\right) \ldots \mu_{n}\left(d a_{n} \mid t_{n}\right) \zeta(d t)$.

The two basic assumptions that Milgrom-Weber make are (a) payoffs are equicontinuous and (b) the information structure is absolutely continuous. Conditions which imply either (a) or (b) are given in [16, p. 625]. Balder has succeeded in generalizing, their results by relaxing (a), but he still needs (b) ? For the proof of Theorem 3.3 we did not make use of any of these assumptions. It is important to note that assumption (b) allows the above authors to express the expected utility (4.1) in a convenient way (see [16, p. 625] or [3]). In particular, once distributional strategies are topologized with weak convergence, strategy sets are compact metric spaces, expected utflity is continuous and linear and therefore the standard results of either Glicksberg, Fan or Browder (see [16] or [3] for a statement of this result) can be directly applied to prove the existence of an equilibrium. We would like to note that in our framework the expected utility is required to be only quasi-concave in each player's own strategy. Moreover, our expected utility is random, i.e., depends on the states of nature of the world. The latter is quite important since with random expected payoffs, Glicksberg's result is not directly applicable and the use of measurable selection theorems is needed.

Although it is not obvious how one from the approach of Milgrom-Weber and Balder can obtain versions of our Theorem 3.3, it is very clear that Theorem 3.3 is not subsumed by any of their results. In particular, no assumption of equicontinuity of payoffs is needed and the set of players is not necessarily finite. It may be instructive to note that our approach, i.e., working with strategles which are measurable functions, seems to be quite natural to analyze economies with incomplete information as recently defined in Palfrey-Srivastava $[19,20]$ and Postlewaite-Schmeidler [21] or uncertainty in market games examined in Peck-Shell [22]. In fact, our approach, 
as well as Theorem 3.3 have been motivated from the work of the above authors. Finally, we would like to note that Mas-Colell [14] viewing a game as a probability measure on the space of utility functions has proved Nash equilibrium existence theorems. He indicates that his existence results may be useful to obtain results for games with incomplete information. 


\section{CONCLUDING REMARKS}

Remark 6.1: We now show how a version of Theorem 3.2 can be easily obtained by combining the deterministic equilibrium result in YannelisPrabhakar [25] with the Aumann measurable selection theorem.

Theorem 6.1: Theorem 3.2 remains true if one replaces assumptions $(a .2 .2)$ and $(a .2 .4)$ by

(a.2.2') $\operatorname{conP}_{1}(\cdot, \cdot)$ is lower measurable, i.e., for every open $v$ in $x_{1}$, the set $\left\{(\omega, x): \operatorname{conP}_{1}(\omega, x) \cap v \neq \phi\right\}$ belongs to $\mathcal{F} \otimes B(x)$.

(a.2.4') For each $\omega \in \Omega, P_{1}(\omega, \cdot)$ has open lower sections, i.e., for each $\omega \in \Omega$ and for each $y_{1} \in x_{1}$, the set $P_{1}^{-1}\left(\omega, y_{1}\right)=\left\{x \in X: y_{1} \in P_{i}(\omega, x)\right\}$ is open in $X$.

Proof: For each $i \in I$ define $\phi_{1}: \Omega \times x+2^{X_{1}}$ by $\phi_{1}(\omega, x)=\operatorname{conP}_{1}(\omega, x)$. By assumption $\left(a .2 .2^{\prime}\right), \phi_{1}\left(\cdot, \cdot^{\circ}\right)$ is lower measurable. Define the correspondence $\Phi: \Omega \times X \rightarrow 2^{X}$ by $\Phi(\omega, x)=\prod_{i \in I_{1}}(\omega, x)$. By. virtue of $(F .2 .6), \Phi(\cdot, \cdot)$ is lower measurable. Define the correspondence $\Gamma: \Omega \rightarrow 2^{X}$ by

$$
\Gamma(\omega)=\{x \in X: \Phi(\omega, x)=\phi\} .
$$

We will show that a measurable selection for $\Gamma(\cdot)$ is a random equilibrium for the random game $E=\left\{\left(X_{1}, P_{1}\right): 1 \in I\right\}$.

In order to apply the Aumann measurable selection theorem (F.2.3), we need to show that $\Gamma(\cdot)$ has a measurable graph and it is nonempty valued. Since $\Phi(\cdot, \cdot)$ is lower measurable, the set

$$
\mathrm{K}=\{(\omega, \mathrm{x}) \in \Omega \times \mathrm{X}: \Phi(\omega, \mathrm{x}) \neq \phi\}=\{(\omega, \mathrm{x}) \in \Omega \times \mathrm{X}: \Phi(\omega, \mathrm{x}) \cap \mathrm{X} \neq \phi\}
$$

belongs to $f \otimes B(x)$ and so does the complement of the set $k$ which is denoted by 
$K^{c}$. Observe now that

$$
\begin{aligned}
G_{\Gamma}=\{(\omega, x) \in \Omega \times x: x \in \Gamma(\omega)\} & =\{(\omega, x) \in \Omega \times x: \Phi(\omega, x)=\phi\} \\
& =\{(\omega, x) \in \Omega \times x: \Phi(\omega, x) \neq \phi\}^{c} \\
& =K^{c},
\end{aligned}
$$

and the latter set belongs to $\mathcal{F} \otimes(x)$ as it was noted above. Therefore, $\Gamma(\cdot)$ has a measurable graph. Moreover, an appeal to Theorem 6.1 in [25, p. 242] (where in [25] for each $i \in I$ and for each $x \in X$ we set $A_{i}(x)=\bar{A}_{i}(x)=x_{i}$ ) shows that for each $\omega \in \Omega, \Gamma(\omega) \neq \phi$. Therefore, by the Aumann measurable selection theorem there exists a measurable function $\mathrm{x}^{*}: \Omega \rightarrow \mathrm{X}$ such that $x^{*}(\omega) \in \Gamma(\omega)$ for almost all $\omega \in \Omega$, i.e., $\Phi\left(\omega, x^{*}(\omega)\right)=\phi$ for almost all $\omega \in \Omega$, which implies that for each $1 \in I, P_{1}\left(\omega, x^{*}(\omega)\right)=\phi$ for almost all $\omega \in \Omega$, i.e., $x^{*}(\cdot)$ is a random equilibrium for $\mathrm{E}$.

Note that assumption $\left(2.2 .4^{\prime}\right)$ of Theorem 6.1 is weaker than assumption (a.2.4) of Theorem 3.2, and, assumption (a.2.2') is different than assumption (a.2.2). Hence, neither result implies the other. However, the methods of proof are different. It can be easily seen that Corollary 3.2 follows directly from Theorem 6.1. The idea of the proof is identical with the one used to prove Corollary 3.1 .

Remark 6.2: The form of the Bayesian game defined in section 3.2 can be generalized by replacing each player's random payoff function $h_{i}: \Omega \times X \rightarrow I R$, by a random preference correspondence $P_{i}: \Omega \times X \rightarrow 2^{X_{i}}$. Following the notation of Section 3.2, in this new setting the conditional expected payoff of each player (denoted by $\Phi_{1}(\omega, x)$ ) becomes now a correspondence from $\Omega \times x$ to 2 . By replacing assumptions (a.3.2) - (a.3.5) with:

(a.3.2') For each fixed $\omega \in \Omega, P_{1}(\omega, \cdot)$ is $1.3 . c .$, 
$\left(a .3 .3^{\prime}\right) \operatorname{con} \Phi_{1}(\cdot, \cdot)$ is lower measurable, 8

(a.3.4') for each measurable function $x: \Omega \rightarrow x, x_{1}(\omega) \notin \operatorname{con} \emptyset(\omega, x(\omega))$ for almost all $\omega \in \Omega$,

(a.3.5') $P_{i}$ is Integrably bounded, and it has a measurable graph,

and invoking to Corollary 5.2 in [26] (which says that the integral of a 1.s.c. correspondence which is integrably bounded and has a measurable graph, is also 1.s.c.) we can guarantee that for each fixed $\omega \in \Omega, \Phi_{1}(\omega, \cdot)$ is 1.s.c. and therefore by appealing to Theorem 3.1 one can prove the existence of a Bayesian equilibrium, for this more general form of a Bayesian game.

Remark 6.3: Theorem 3.3 remains true if assumption (a.3.1) is replaced by:

(a.3.1') $x_{i}$ is a compact, convex, nonempty subset of a separable Banach space.

The proof of Theorem 3.3 remalns the same, one just needs to appeal to Corollary 3.2 instead of Corollary 3.1 . 
FOOTNOTES

1. Notice that this notion of equilibrium is non-cooperative. No communication between players is allowed.

2. However, no equilibrium existence results are contained in the above papers. Balder [3], Mas-Colel1 [14], Milgrom-Weber [16] and RadnerRosenthal [23] have provided existence of equilibrium theorems for games with incomplete information, but their approach is different than ours. We will discuss the work of the above authors in Section 5.

3. An alternative proof of a version of Theorem 3.2 will be given in Section 6 .

4. If $\phi: T+2^{X}$ is a correspondence from a measurable space $T$ to a Banach space $X$, we say that $\phi$ is integrably bounded if the re exists an integrable function $g: T \rightarrow \mathbb{R}$ such that $\sup \{\|x\|: x \in \phi(t)\} \leqslant g(t)$ for almost all $t$ in $T$.

5. Note that the proofs of Theorem 3.1 and Corollary 3.1 remain unchanged if the measurability assumptions on either the preference correspondence $P_{i}$ or the payoff function $u_{i}$ of each player are made with respect to the partition $s_{i}$ instead of $\mathcal{Z}$.

6. Since the connection between [16] and [23] has already been discussed by Milgrom-Weber elsewhere (see [16] for an exact reference), we will focus on the mixed strategy equilibrium existence results given in [3] and [16].

7. It should also be mentioned that Balder does not impose any topological structure on the type spaces $T_{1}$.

8. I.e., for every open subset $v$ of $x_{1}$ the $\operatorname{set}\left\{(\omega, x): \operatorname{con} \Phi_{1}(\omega, x) \cap V \neq \phi\right\}$ belongs to $s_{1} \otimes B(x)$. 


\section{REFERENCES}

[1] Arrow, K. and G. Debreu, "Existence of an Equilibrium for a Competitive Economy," Econometrica 22, 1954, 265-290.

[2] Aumann, R. J., "Correlated Equilibrium as an Expression of Bayesian Rationality," Econometrica 55, 1987, 1-18.

[3] Balder, E. J., "Generalized Equilibrium Results for Games with Incomplete Information," Mathematics of Operations Research, (to appear).

[4] Browder, F. E., "The Fixed Point Theory of Multi-valued Mappings in Topological Vector Spaces," Mathematische Annalen 177, 1968, 283-301.

[5] Castaing, C. and M. Valadier, Convex Analysis and Measurable Multifunctions, Lecture Notes in Mathematics, No. 580, Springer-Verlag, New York, 1977.

[6] Debreu, G., "A Social Equilibrium Existence Theorem," Proceedings of the National Academy of Sciences, U.S.A. 38, 1957, 886-893.

[7] Fan., K., "Fixed-Point and Minimax Theorems in Locally Convex Topological Linear Spaces," Proceedings of the National Academy of Sciences, U.S.A. $38,1952,121-126$.

[8] Fan, K., "Extensions of Two Fixed Point Theorems of F. E. Browder," Mathematische Zeitschrift $112,1969,234-240$.

[9] Fan, K., "Applications of a Theorem Concerning Sets with Convex Sections," Mathematische Annalen $163,1966,189-203$.

[10] Harsany1, J. C., "Games with Incomplete Information played by Bayesian Players," Parts I, II, III, Management Sclence 14, 1967, 159-182, 320$334,486-502$.

[11] Himmelberg, C. J., "Measurable Relations," Fundamenta Mathematicae LXXXVII, $1975,53-72$. 
[12] Kim, T., K. Prikry and N. C. Yannelis, "Caratheodory-Type Selections and Random Fixed Point Theorems," Journal of Mathematical Analysis and Applications, $122,1987,383-407$.

[13] Kim, T., K. Prikry and N. C. Yannelis, "On a Caratheodory-Type Selection Theorem," Journal of Mathematical Analysis and Applications, (to appear).

[14] Mas-Cole1l, A., "On a Theorem of Schmeidler," Journal of Mathematical Economics 13, 1984, 201-206.

[15] Michael, E., "Continuous Selections I," Annals of Mathematics 63, $1956,361-382$.

[16] Milgrom, P. and R. Weber, "Distributional Strategies for Games with Incomplete Information, Mathematics of Operations Research 10, 1985, 619-632.

[17] Myerson, R., "Bayesian Equilibrium and Incentive Compatibility: An Introduction," Kellogg Graduate School of Management, Northwestern University, Discussion Paper No. 548, 1983.

[18] Nash, J., "Non-Cooperative Games," Annals of Mathematics 54, 1951, 286-295.

[19] Palfrey, T. and S. Srivastava, "Private Information in Large Economies," Journal of Economic Theory 39, 1986, 34-58.

[20] Palfrey, T. and S. Srivastava, "On Bayesian Implementable Allocations," Review of Economic Studies LIV, 1987, 193-208.

[21] Peck, J. and K. She11, "Market Uncertainty: Correlated Equilibrium and Sunspot Equilibrium in Imperfectly Competitive Economies," Department of Economics, Cornell University, 1987, (mimeo).

[22] Postlewaite, A. and D. Schmeldler, "Implementation in Differential Information Economies," Journal of Economic Theory 39, 1986, 14-33.

[23] Radner, R. and R. Rosenthal, "Private Information and Pure-Strategy Equilibria," Mathematics of Operations Research 7, 1982, 401-409. 
[24] Shafer, W. and H. Sonnenschein, "Equilibrium in Abstract Economies without Ordered Preferences," Journal of Mathematical Economics 2, $1975,345-348$.

[25] Yannelis, N. C. and N. D. Prabhakar, "Existence of Maximal Elements and Equilibria in Linear Topological Spaces," Journal of Mathematical Economics $12,1983,233-245$.

[26] Yannelis, N. C., "On the Lebesgue-Aumann Dominated Convergence Theorem in Infinite Dimensional Spaces," 1987, (mimeo). 